\title{
Management of Failed Proximal Biceps Surgery: Clinical Outcomes after Revision to Subpectoral Biceps Tenodesis
}

\author{
David Savin, MD1, Brian Robert Waterman, MD², Catherine Richardson ${ }^{3}$, Shelby Sumner ${ }^{4}$, Anthony A. Romeo, MD ${ }^{5}$ \\ ${ }^{1}$ Desert Orthopedic Center, Rancho Mirage, CA, USA, ${ }^{2}$ Wake Forest University School of Medicine, Winston Salem, NC, USA, \\ ${ }^{3}$ Rush University, Chicago, IL, USA, ${ }^{4}$ Rush University Medical Center - Chicago, IL, Chicago, IL, USA, ${ }^{5}$ Midwest Orthopaedics at \\ Rush, Chicago, IL, USA.
}

Objectives: The preferred technique for management of biceps-superior labral pathology is often debated, and rates of revision and persistence pain vary widely accordingly to surgical technique and patient demographics. The purpose of this study was to evaluate the clinical and functional outcomes of patients undergoing revision subpectoral biceps tenodesis after failed primary tenodesis or tenotomy.

Methods: A retrospective review was completed to identify of all patients undergoing revision biceps tenodesis by the senior surgeon with minimum 24-month follow-up. Demographic variables including age, sex, insurance status, and tobacco use were recorded, Patient reported outcomes including the functional score, Single Assessment Mumeric Evaluation (SANE) rating, Visual Analog Scale (VAS) pain scale, Simple Shoulder Test (SST), and American Shoulder and Elbow Score (ASES) were obtained, and range of motion (ROM), strength, and complications were quantified.

Results: In total, 36 patients with revision biceps tenodesis were identified, with a mean age of $46 \pm 12$ years and mean follow up of $60 \pm 29$ months. The indication for revision surgery was failure of index suprapectoral biceps tenodesis $(56 \%)$, subpectoral biceps tenodesis $(36 \%)$, and failure of tenotomy $(8 \%)$. Concomitant procedures including rotator cuff repair and capsular release were performed in $25 \%$ and $8 \%$ of index surgeries respectively. There was a significant improvement in VAS score $(P<0.001)$, SST $(P<0.009)$, functional score $(P<0.001)$ and forward elevation $(P<0.001)$. Postoperative shoulder strength $(P=0.082)$, SANE $(P=0.074)$, abduction $(P=0.096)$ and external rotation $(P=0.8)$ improved, but failed to achieve statistical significance. were not significantly improved following revision surgery. There was no difference in post-operative outcome measures between surgical indication, concomitant procedures and sex. $86 \%$ of patients reported high satisfaction and stated they would have this revision surgery again. Overall complication rate was $33 \%$ with the majority of these being either acute or chronic pain, with $8 \%$ of patients requiring additional surgeries to manage stiffness, chronic pain or rotator cuff deficiency.

Conclusion: The current study demonstrates high patient satisfaction $(86 \%)$ and significant improvement in functional outcomes with revision biceps tenodesis after previous failed tenodesis or tenotomy. Revision biceps tenodesis using a mini-open, subpectoral technique may be an effective strategy to address failed prior surgery, although the potential for persistent pain must be emphasized.

The Orthopaedic Journal of Sports Medicine, 6(7)(suppl 4)

DOI: $10.1177 / 2325967118 S 00106$

CThe Author(s) 2018 